\title{
PLC and SCADA Laboratory Experiments for a Final Year Instrumentation Course
}

\author{
Kiing Ing Wong and Teck Ung Siaw
}

\begin{abstract}
This paper presents a method of preparing Programmer Logic Controller (PLC) and Supervisory and Data Acquisition (SCADA) laboratory experiments for a final year instrumentation course. It is the process of continuous quality improvement to produce work-ready graduates. The students are taught with the up to date technology for monitoring and control using in industry. Recently, Ethernet-enable PLC was rapidly introduced into modern SCADA systems for the monitoring and control of industrial process, other scientific application and even office processes. However, set up a modern SCADA laboratory may sometimes costly and requires specific skills. Therefore, institutes and instructors may reluctant to do it. In this paper, it will be shown that generally the PLC and SCADA laboratory experiments can be set up within limited budget and the student can completed the basic experiments in four laboratory sections in one semester period.
\end{abstract}

Index Terms-PLC, SCADA, teaching and learning.

\section{INTRODUCTION}

Instrumentation covers a wide range of topics from electric circuit, signal and systems, electronic, sensor technology, data communication, dynamic and control theories, to digital signal processing and software engineering and design. It is vital to the monitoring and control of distributed industrial systems, like electrical power generation, transmission and distribution, oil and gas production and processing, water management, car manufacturing and many others. Therefore, students gain insight to the subject will enable them to explore many job opportunities when they graduate from the university.

For the final year electronic and electrical engineering students, instrumentation course shall covers deeper into specific topics on PLC and SCADA because they have learned the basic theories of electric circuit, electronic and signal processing. Since instrumentation is a huge topic, some instructors may prepare laboratory experiments based on their experience and knowledge. For example, instructors from the electronic design background may prepare sensory and microcontroller laboratories. These types of laboratories will spend up to four laboratory section (or one semester period). If the instrumentation course is offered to the final year electrical and electronic engineering students, the students may have done almost similar laboratory exercises

Manuscript received May 2, 2014; revised July 31, 2014. The conference attendance was support in part by the teaching and learning department of Curtin University, Malaysia.

K. I. Wong and T. U. Siaw are with the Electrical and Computer Engineering Department, Curtin University, Sarawak, CO 98000 Malaysia (e-mail: wong.kiing.ing@curtin.edu.my, teck_ung@hotmail.com). in their junior years in other courses, such as electrical circuit and electronic. Therefore, the students will miss the opportunities to learn the basic skills to monitor and control in practical environment.

Since 2000, every engineering programme in Malaysia has to be accredited by the Engineering Accreditation Council (EAC) [1]. If the engineering programme is not been accredited, the graduate will not able to register with Board of Engineering, Malaysia (BEM) that allows them to practice engineering in graduate level. Therefore, the university and department began the process of documenting how program outcomes (PO) would be achieved, and the lecturers started to think about how might units at the university be transformed so that it genuinely impacts the PO [2], [3]. We set up the action plans for continuous quality improvement in the instrumentation course for final year students. In the action plan, we decided to increase the percentage of the assessment to more advanced laboratory works relating to PLC and SCADA.

This paper includes the descriptions of a modern SCADA-based industrial control system. The details on how to set up a cost effective PLC and SCADA laboratories are proposed.

\section{MODERn MONITORING AND CONTROL SySTEM}

Nowadays, most production processes are computerized in order to more efficiently monitor and control the field equipment, using a SCADA system. SCADA has advanced considerably over the last years in line with the technological advances of information technology (IT). The current trend is to extend the existing SCADA system to the Internet, for larger and wide-area system monitoring and control [4], [5].

Fig. 1 illustrates a simple example of a modern SCADA-based process control application that can be found in a more complicated system in food and chemical processing. The SCADA system is generally located in a remote location that allows the operator to check the statues of the processes and the variables of field equipment are appropriately. The SCADA also is able to record the volume of the production. In the Fig. 1, the volume of the production can be calculate by the number of time the output valve is opened (to discharge the liquid inside the tank).

Ethernet is used for communication between the SCADA system and the PLC module. There are many different types of communication protocols available for an industrial control network, generally referred as fieldbus protocols. Ethernet fieldbus become more common due to greater reliance of Internet system [4], [5].

Unlike SCADA system that generally used as a supervisory scheme, PLC modules perform field control, 
such as a water tank controller. There are three output and two input devices that are connected to the PLC as shown in Table I. The inlet valve and output valve are used to channel the liquid into and out from the tank, respectively. The full sensor and empty sensors are used to detect the liquid level in the tank. The PLC read the full sensor and empty sensor statues and feed the statues to a controller to determine whether to turn on or off the inlet and outlet valves or to run the motor.
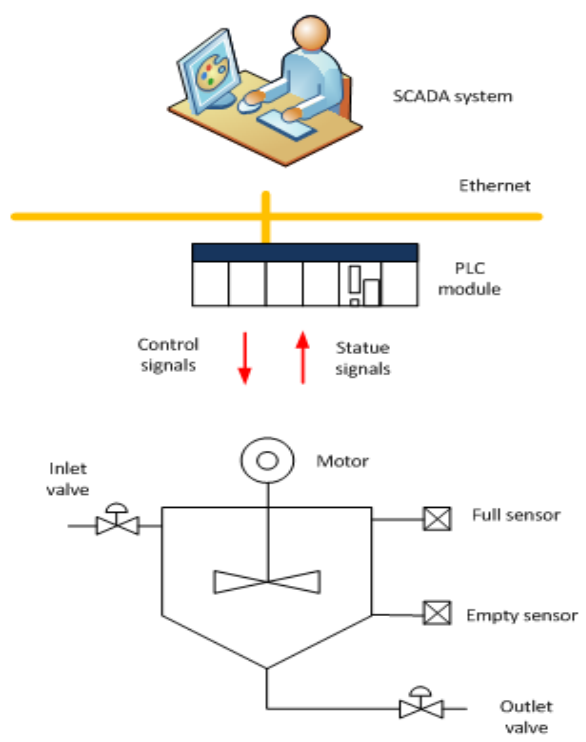

Fig. 1. SCADA-based process control application.

TABLE I: INPUT AND OUTPUT OF PLC

\begin{tabular}{|l|l|}
\hline Output & Input \\
\hline Motor & Full sensor \\
\hline Inlet valve & Empty sensor \\
\hline Outlet valve & \\
\hline
\end{tabular}

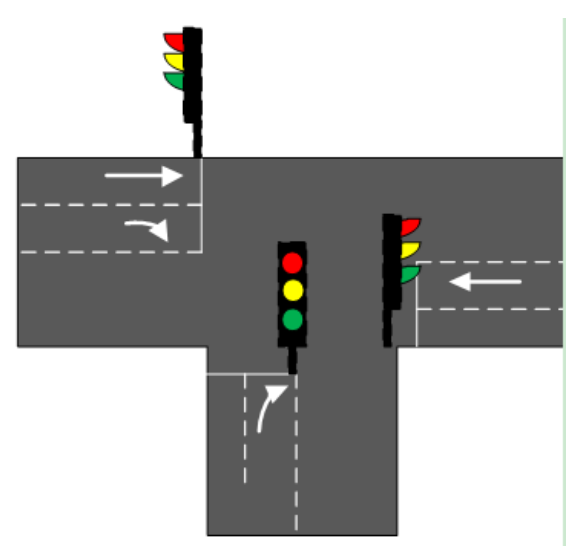

Fig. 2. T- junction traffic light.

\section{PLC}

Students are briefed on what is PLC and how to program PLC using Ladder Diagram during the lecture. Teaching PLC without any laboratory exercise does not mean anything. Therefore, two laboratory sessions are allocated to the students to accomplish some of the tasks. OMRON-CJ1M PLC module was used in the lab.

\section{A. Lab 1}

By completion of Lab 1, students will familiar with how to use the development environment, specifically CX-Programmer from Omron, for developing the program. Students will also able to input signal from the external devices, such as switches and sensors, and output instruction to actuator and lighting. Besides that, students will practice how to create Ladder Diagram logic and use timer and counter functionalities in their designs.

\section{B. $L a b 2$}

Before students came to their second laboratory section, they need to come out with a more advance controller. There are many controllers that are suitable and can complete in one section laboratory time. For example, students may ask to design a T-junction traffic light controller. Fig. 2 shows a $\mathrm{T}$-junction traffic light that consisted of night light indictors. Students are required to describe what does the controller should do, they may use state diagram to effectively represent the state of the controller. Then, the students are able to implement the controller in Ladder Diagram.

\section{SCADA}

Most of the SCADA software development company does not support education and the cost of SCADA software is very high [6]. The hardware and software of the connecting devices (including the PC and the PLC) must be compatible. There are hidden cost imposed when the SCADA are required to be upgrade. Therefore, any commercial SCADA license are not economical viable for universities, especially with small student numbers and tight budget. We used InduSoft Web Studio (ISW) for our SCADA laboratory experiments it provides a free educational license.

Students will be taught on how to configure the setting between PLC and IWS through Wireless Ethernet and also how to create a simple Graphical User Interface (GUI) to simulating the real industrial environment in the first laboratory session. In the second laboratory section, the students are allowed to present their own SCADA design using the skills they learned in the first laboratory section.

\section{A. $\operatorname{Lab} 3$}

A SCADA laboratory activity is designed so that they can complete it in two hours. By completion of the Lab 3, the students will understand the basic working knowledge of IWS and familiar with how to build a small project, configure dynamic graphics and run a simple project for controlling a water tank in a plant.

Before students are running their project, they are required to setup the environment. This includes setting up the Wireless Ethernet connection (WLAN). Students are required to configure both a PLC module which the project will communicate with and a SCADA Windows us to run the project. The lab manual provides step by step on how to configure the PLC in CX-Programmer and the SCADA in IWS using OMETH driver. Some of the steps may not make senses to most of the students at first, what they are required are to move to the next step. The students will understand more when they revisit the procedure in the next lab.

The OMETH driver enables communication between IWS and Omron devices using FINS commands with CS1 or CV 
modes. IWS cannot directly use to program PLC. Some configuration in OMETH driver with the correct IP address needs to be set accordingly with the PLC itself. Fig. 3 shows the main driver sheet for OMETH in IWS and how to configure the station for the PLC.

For TCP/IP Communication [7]:

$\langle$ IPAddress $>:<$ PortNumber $>:<$ Network $>:<$ Node Address $>$

For example, 192.168.1.30:9600:0:46 or 192.168.1.30:9600:0:30 - (depend on the IP address and node address of your PLC)

where:

- $\quad$ IP Address > is the IP address of the device on your TCP/IP network;

- <Port Number> is the communication port;

- <Network Address > is the device's subnet address, according to the FINS protocol;

- <Node Number> is the device's node number, according to the FINS protocol. If you do not specify a value, then Studio will automatically insert the last-used node number.

The I/O Address field in Table II used to associate each tag to its respective device address. Type an address into the Address field that complies with the following syntax:

\section{$<$ Area $>:<$ Format $><$ Address $>$}

For example, CIO: 1.1(PLC output) or CIO: 0.1(PLC input)

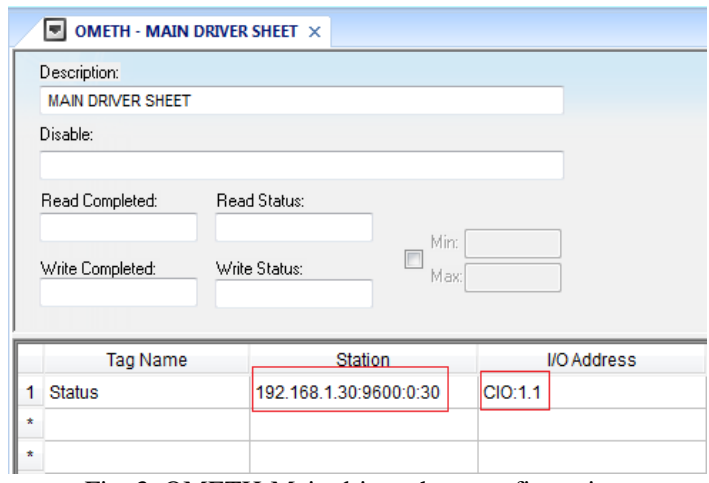

Fig. 3. OMETH-Main driver sheet configuration.

where:

- <Area $>$ is the Memory area (e.g., CIO, WR, HR, AR, and so forth).

- <Format> defines the format of the register. This is an optional parameter; if this field is blank, Studio provides the data in Unsigned Word format.

After the project initialization, the students will guide through how to configure Tags. Variable Tags are a core component in SCADA. It provides the link between the operator and the real-work. Students will create several tags to represent the main GUI control, and the tags to on/off the output switches of a PLC.

Fig. 4 shows the main GUI for the water tank controller, manual controller screen and auto controller screen. In the main GUI, the operator is allowed to assess to the manual and auto controller screens, and trend and alarm screens. In the manual screen, the operator will be granted to full control over the water tank: on/off the inlet and outlet valve and run the motor as shown in Fig. 5 (a). In the real-world scenario, the operators are seldom assesses to the manual mode to take control over the equipment in the plant. When the students turn on the inlet valve in the manual controller screen, the remote PLC output will light out, to indicate output on, as shown in Fig. 6.

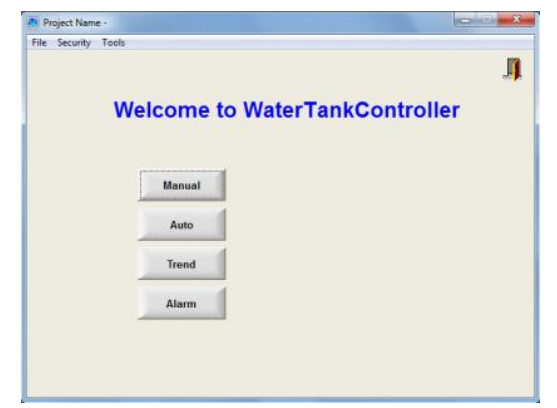

Fig. 4. The main GUI of the water tank controller.

\begin{tabular}{|c|c|c|c|}
\hline Data Types & Sample Syntax & $\begin{array}{l}\text { Valid Range of Initial } \\
\text { Addresses per Worksheet }\end{array}$ & Comments \\
\hline ClO Area & CIO $: 10$ & 0 to 6143 & $\mathrm{ClO}$ area \\
\hline WR Area & WR : 0 & 0 to 511 & Work area \\
\hline HR Area & $\mathrm{HR}: 100$ & $a$ to 511 & Holding bit area \\
\hline AR Area & AR: 135 & 0 to 959 & Auxiliary area \\
\hline DM Area & DM $: 30000$ & 0 to 32767 & DM area \\
\hline Timer Area & TA $: 50$ & 0 to 4095 & Timer area \\
\hline Counter Area & $\mathrm{CA}: 34$ & 0 to 4095 & Counter area \\
\hline EM\# & EM $4: 287$ & 0 to 32767 & $\begin{array}{l}\text { Extended memory area, } \\
\text { where \# is the block no. }\end{array}$ \\
\hline
\end{tabular}

(a)

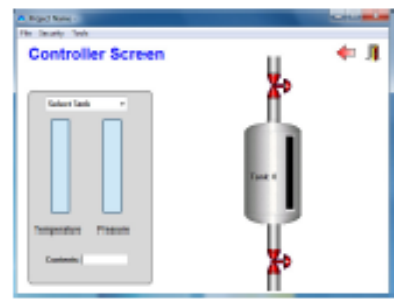

(b)
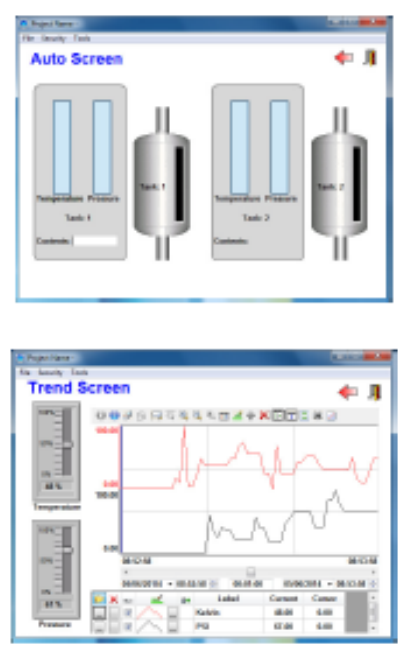

(d)

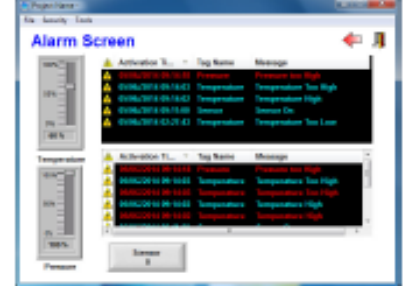

Fig. 5. (a) Manual controller screen, (b) Auto controller screen, (c) Trend controller screen and (d) Alarm controller screen. 
In the auto controller screen in Fig. 5 (b), the operator is able to monitor the statues of the water tank controller in the field. The PLC is executing the water tank controller and reporting sensor variables back to the SCADA system.

\section{B. $L a b 4$}

Before students came to their second SCADA laboratory section, they need to come out with a simple idea of a modern SCADA system. The SCADA system must be completed within one section laboratory time. Students from electrical power majors, many apply the SCADA system to a substation protection system. In their design, they need to include the trend and alarm functionalities.

Fig. 5 (c) shows the example of the trend screen created by the students. The trend screen allows data logging of sensor variables. Since the temperature and pressure sensors are not available at the moment, the students simulate the sensor using the two sliding bars as one is for temperature and the other is for the pressure. The sensor reports to the SCADA system at a rate of one sample every second.

Fig. 5 (d) shows the example of the alarm controller screen created by the student. This screen displays the status of the water tank in term of those selected sensitive parameters such as temperature too high, pressure too low and etc. If the temperature is too high, the system will alert the alarm and the operator will get noticed in order to let the treatment process to take place in time. All this data was saved and store for future reference.
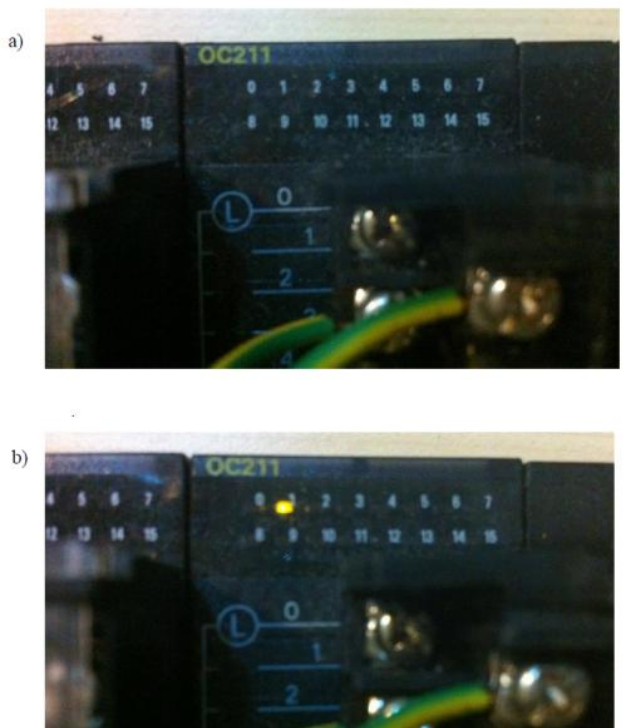

Fig. 6. (a) Before the inlet valve is turned on and (b) after inlet valve is turn on.

\section{CONCLUSION}

It was observed that students were interested to learn more about PLC and SCADA because they found it is relevant to the real-world applications and useful for them to final a job in future. Once the students understand the topics of PLC and able to design the controller based on Ladder Diagram, most of them did not have any problem in implementing the controller on the Omron PLC module, using CX-Programmer. However, they did found it difficult to initialize the SCADA project for communication with a PLC (through Ethernet) in this first time. But, they were able to grasp the correct concepts when they repeat the procedure in their second SCADA laboratory section.

Since 2013, we changed our SCADA software to ISW from InduSoft. We found that ISW with free education license is very useful for the teaching purposes. Previously, we used the free trail SCADA software. It has all the features of the standard SCADA software, except it does not support any physical connection with a physical device, such as a PLC module. Students found it uninteresting, because the instrumentation communication protocols, such as Ethernet, could not be tested in the laboratory. With the free education license ISW, students would able to instruct commands from a SCADA computer to a remote PLC module, through Ethernet connection.

\section{REFERENCES}

[1] Engineering Accreditation Council (EAC) Malaysia. [Online]. Available: http://www.eac.org.my/web/about_EAC.html.

[2] S. Danielson and B. Rogers, "A methodology for direct assessment of student attainment of program outcomes," American Society for Engineering Education, 2007.

[3] Z. T. Deng, R. Rojas-Oviedo, and X. Qian, "Developing assessment tool for outcome based engineering courses," in Proc. the 2002 American Society for Engineering Annual Conf. \& Exposition, Session 1566, 2002.

[4] B. Galloway and G. P. Hancke, "Introduction to industrial control network," IEEE Communications Surveys \& Tutorials, vol. 15, no. 2, pp. 860-880, 2013

[5] D. J. Kang, J. J. Lee, S. J. Kim, and K. H. Park, "Analysis on cyber threats to SCADA systems," Transmission \& Distribution Conf. \& Exposition: Asia and Pacific, pp. 26-30, 2009.

[6] Z. Zedic and A. Zafalski, "Teaching mechatronics using SCADA," in Proc. $4^{\text {th }}$ WIETE Annu. Conf. Engineering and Technology, pp. 69-72, 2013.

[7] OMETH Communication Driver: Driver for Serial and Ethernet Communication with Omron Devices Using FINS commands, Omron PLC, Version 10.5, Doc. Revision R, 2011.

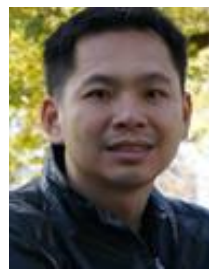

Kiing Ing Wong was born in Sarawak, Malaysia in 1975. He received the B.Eng. (Hons) and Ph.D. degrees in electrical engineering from the University of Manchester in 1999 and University of Southampton in 2004 , respectively. He taught in the Southern Taiwan University of Technology for two semesters before he joined Curtin University in 2005 as a Lecturer. He was promoted to senior lecturer in 2008.

Dr. Kiing Ing Wong research interests include wearable, ergonomic body sensor design and effective teaching using SCADA.

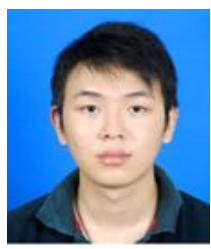

Teck Ung Siaw was born in Sarawak, Malaysia in 1989. He currently is pursuing an undergraduate degree in electrical power engineering at Curtin University, Sarawak Malaysia. His research interests include renewable energy for off-grid Sarawak community, and effective teaching using SCADA. 